\title{
Brief RU 38486 Treatment Normalizes the Effects of Chronic Stress on Calcium Currents in Rat Hippocampal CAI Neurons
}

\author{
Henk Karst*,', and Marian Joëls' \\ 'SILS - CNS, University of Amsterdam, Amsterdam, The Netherlands
}

\begin{abstract}
Chronic stress alters many properties in rat brain, like serotonin responsiveness and dendritic morphology. In the present study, we examined (i) whether unpredictable stress during 21 days affects calcium (Ca) currents of CAI pyramidal neurons recorded on day 22; and (ii) if so, whether this change is normalized by treatment with the glucocorticoid receptor-antagonist RU 38486 during days I8-2I. At 3 weeks of unpredictable stress increased the amplitude of the peak and sustained calcium current components, determined in hippocampal slices prepared from animals under rest (ie, with low corticosterone levels). The increased Ca-current amplitude was associated with an enhanced cell capacitance; current density was not significantly affected by chronic stress. In slices from stressed rats that received RU 38486, no stress-induced enhancement of calcium current amplitude was seen, while RU 38486 by itself did not alter calcium currents in handled controls. We confirmed earlier observations that brief in vitro treatment with $100 \mathrm{nM}$ corticosterone, thus substantially activating the low-affinity glucocorticoid receptors, increases Ca-current amplitude recorded I-4h later in slices from naïve rats. However, Ca-current amplitude was not affected by corticosterone applied to slices from handled controls and currents were even decreased by corticosterone given to slices from chronically stressed rats, suggesting that corticosterone effects depend on the history of the animal. In conclusion, the data indicate that chronic stress, RU 38486 treatment as well as acute rises in corticosterone level strongly modulate calcium influx into CAI neurons. This could have consequences for the viability of these neurons.

Neuropsychopharmacology (2007) 32, I830- 1839; doi: I0.1038/sj.npp. I 301296; published online 17 January 2007
\end{abstract}

Keywords: unpredictable stress; glucocorticoid receptor; capacitance; current amplitude; whole cell recording

\section{INTRODUCTION}

Exposure of rats to a stressful situation activates the hypothalamo-pituitary-adrenal (HPA) axis, resulting in enhanced release of corticosterone (McEwen and Wingfield, 2003; De Kloet et al, 2005). Corticosterone affects peripheral organs but also enters the brain, where it binds to intracellular receptors and alters transcription of responsive genes. Low levels of corticosterone, circulating under rest at the circadian trough, are sufficient to substantially activate high-affinity mineralocorticoid receptors (MRs), whereas lower affinity glucocorticoid receptors (GRs) only become fully activated after stress or at the circadian peak (Reul and de Kloet, 1985).

Pyramidal neurons in the CA1 hippocampal area express both MRs and GRs (Van Steensel et al, 1996). Differential activation of these receptors alters several properties of

* Correspondence: Dr H Karst, SILS-CNS, University of Amsterdam, Kruislaan 320, 1098 SM Amsterdam, The Netherlands, Tel: + 3120 5257647, Fax: + 3120 5257709, E-mail: karst@science.uva.nl Received 6 July 2006; revised 5 October 2006; accepted 30 October 2006 pyramidal neurons (Joëls, 2001). Influx of calcium (Ca) via voltage-gated ion channels for example is very sensitive to $M R$ and GR activation: Predominant MR activation is associated with restricted Ca-influx (Karst et al, 1994; Nair et al, 1998; Joëls et al, 2003). After stress, that is when GRs become activated, Ca-current amplitude increases, with a delay of several hours (Kerr et al, 1992; Karst et al, 1994; Joëls et al, 2003). The increased Ca-influx is due to DNAbinding of GR homodimers (Karst et al, 2000).

In addition to acute stress, chronic stress - caused by prolonged exposure to physical stressors or by psychosocial factors - also largely alters properties of rat brain cells, such as dendritic morphology and neurogenesis, leading to changes in behavioral performance (for reviews McEwen, 1999; McEwen and Chattarji, 2004; Conrad, 2006; Czeh et al, 2001). Some of these alterations were also seen with a milder paradigm, where rats are exposed to an unpredictable sequence of mild stressors (Chappell et al, 1986; Ni et al, 1999; Cullinan and Wolfe, 2000; Vyas et al, 2002; Sergeyev et al, 2005). This paradigm clearly affects the function of CA1 neurons (Joëls et al, 2004). For instance, long-term potentiation is impaired after chronic unpredictable stress (Alfarez et al, 2003). Likewise, responses of CA1 pyramidal 
cells to serotonin are attenuated (Van Riel et al, 2003). This is of interest since chronically stressful situations can be a risk-factor for the onset of major depression (Holsboer, 2000; Brown et al, 2004; Nemeroff and Vale, 2005; Berton and Nestler, 2006). Thus, elevated hormone levels are seen prior to any clinical symptoms in high-risk proband (Ising et al, 2005). Also, the normalization of hormone levels upon treatment predicts for relapse probability (Ribeiro et al, 1993; Zobel et al, 2001). Finally, patients with bipolar or psychotic depression show improvement of their symptoms already within a week of treatment with the GR-antagonist RU 38486 (Belanoff et al, 2001; Young et al, 2004; Simpson et al, 2005; Flores et al, 2006).

Despite clear effects of acute stress on CA1 Ca-currents, no studies so far have examined the susceptibility of these currents to chronic stress. Therefore, we here investigated the effects of 21 days of unpredictable stress on Ca-current characteristics in rat CA1 pyramidal neurons and compared these with Ca-currents in handled controls. Currents were studied on day 22, under conditions of predominant MR activation or concurrent activation of MRs and GRs, that is $1-4 \mathrm{~h}$ after brief in vitro corticosterone administration (100 nM; Karst et al, 2000). To examine the potential of a GR-antagonist to normalize stressinduced effects, half of the animals were treated during days 18-21 of the stress protocol with a high dose of RU 38486 twice daily.

\section{MATERIALS AND METHODS}

\section{Animals and Stress Protocol}

Male Wistar rats (Harlan CPB, The Netherlands; $175-250 \mathrm{~g}$ at the start of the procedure) were housed in pairs, from at least 7 days before the experimental procedures started. Food and water were provided ad libitum, lights were on between 0800 and 2000 and temperature and humidity were kept around $20-22^{\circ} \mathrm{C}$ and $55 \%$, respectively. All experiments were carried out in accordance with the Declaration of Helsinki and approved by the local committee on Animal Bioethics of the University of Amsterdam.

At the start of the experiment, rats were randomly assigned to either the handled control (handled, $n=48$ ) or chronic unpredictable stress (stress, $n=48$ ) groups. Control rats were removed from their cages, handled briefly and weighed, for 21 days. The other rats were exposed for a 21days period to (different) stressors twice daily in a random order (Chappell et al, 1986; Herman et al, 1995): (1) Immobilization: rats were placed in plastic immobilization cages for $1 \mathrm{~h}$; (2) Cold immobilization: rats were placed in plastic immobilization cages in a cold room $\left(4^{\circ} \mathrm{C}\right)$ for $1 \mathrm{~h}$; (3) Vibration: rats in group-cages were placed on top of an orbital shaker and shaken for $1 \mathrm{~h}$ at $30 \mathrm{rev} / \mathrm{min}$; (4) Isolation: rats were moved to individual cages for a $24-\mathrm{h}$ period; (5) Crowding: rats were housed four to six per cage for a 24-h period; (6) Swim: rats were placed in a plastic container filled with water $\left(25-30^{\circ} \mathrm{C}\right)$ for $30 \mathrm{~min}$; (7) Coldwater swim: rats were placed in a plastic container filled with cold water $\left(10-15^{\circ} \mathrm{C}\right)$ for $5 \mathrm{~min}$. Rats were exposed to cold only once per day.

During the last 4 days of the 21 days period, half of the handled and the stressed animals received RU 38486
( $5 \mathrm{mg} / 100 \mathrm{mg}$ body weight dissolved in $1.5 \mathrm{ml}$ milk) via a gastro-oesophageal tube directly into the stomach (Karst et al, 1997a); the other half of the animals only received milk $(1.5 \mathrm{ml})$. RU 38486 and milk were given twice daily, just before the handling or stress exposure. On day 22, rats were killed between 9 and $10 \mathrm{am}$ when plasma corticosterone levels are low. One rat of each pair that was killed on a particular day was used for electrophysiological recordings, the other rat was cardially perfused for later histology. We here only report on the former group of animals (48 in total, $n=12$ per experimental group). Trunk blood was collected for plasma corticosterone level analysis by means of a radio immunoassay. Adrenals and thymus gland were collected, cleaned, and weighed.

In addition to these handled and stressed animals, we also examined four naïve rats of a weight (175-250 g at the start of the experiment) that was comparable to the handled group. These rats were group-housed for 22 days, but not subjected to daily handling nor to a 4-days gastrooesophageal administration of milk at days 18-21.

\section{Slice Preparation}

Immediately after decapitation, the brain was removed from the skull and kept in artificial cerebrospinal fluid (aCSF; $4^{\circ} \mathrm{C}$ ) containing (in mmol/l): $\mathrm{NaCl} \mathrm{120,} \mathrm{KCl} 3.5, \mathrm{MgSO}_{4} 5.0$, $\mathrm{NaH}_{2} \mathrm{PO}_{4} 1.25, \mathrm{CaCl}_{2}$ 0.2, D-glucose 10, and $\mathrm{NaHCO}_{3}$ 25.0, gassed with $95 \% \mathrm{O}_{2}$ and $5 \% \mathrm{CO}_{2}$. Next, hippocampal slices $(400 \mu \mathrm{m})$ were prepared with a vibroslicer (Leica VT 1000S, Germany). Frontal lobes and cerebellum were removed and the frontal side of the brain was glued on the slicing plateau. Coronal slices were prepared and incubated at room temperature in recording aCSF containing (in $\mathrm{mmol} / \mathrm{l}$ ): $\mathrm{NaCl} 120, \mathrm{KCl} 3.5, \mathrm{MgSO}_{4}$ 1.3, $\mathrm{NaH}_{2} \mathrm{PO}_{4}$ 1.25, $\mathrm{CaCl}_{2}$ 2.5, D-glucose 10 , and $\mathrm{NaHCO}_{3} 25$. After $1 \mathrm{~h}$, half of the slices from all experimental groups were treated with corticosterone ( $100 \mathrm{nM}$ in $0.01 \%$ ethanol) for $20 \mathrm{~min}$ at $32^{\circ} \mathrm{C}$, the other half with vehicle in aCSF. All cells were recorded $1-4 \mathrm{~h}$ after corticosterone or vehicle treatment, to allow gene-mediated effects to develop. Previous studies have shown that this concentration and delay are sufficient to activate GRs in vitro (Karst et al, 2000).

\section{Current Recording}

Calcium currents were recorded from CA1 pyramidal neurons, using the in situ patch clamp technique. During recording tetrodotoxin $(0.5 \mu \mathrm{M})$, tetraethylammonium-Cl $(10 \mathrm{mM}), 4$-aminopyridine $(5 \mathrm{mM})$ and $\mathrm{CsCl}(5 \mathrm{mM})$ were added to the ACSF to block sodium and potassium channels, respectively. This solution had a $\mathrm{pH}$ of 7.4 and osmolality of $305 \mathrm{mOsm}$ (osmolality determined with a Wescor Inc. 5100C vapor pressure osmometer).

An upright microscope (Nikon 104 Optiphot) with waterimmersion objective $(\times 40)$ and $\times 10$ ocular was used to visualize the neurons. Patch pipettes for recording $(1.5 \mathrm{~mm}$ outer diameter, borosilicate glass; impedance 3-4 M $\Omega$ ) were pulled on a Sutter micropipette puller and filled with: Cs-methane sulfonate (141 mM), HEPES $(10 \mathrm{mM})$, BAPTA $(5 \mathrm{mM})$, MgATP $(2 \mathrm{mM})$, NaGTP $(0.1 \mathrm{mM}) ; \mathrm{pH} 7.4,295 \mathrm{mOsm}$. This solution is very suitable 
to record high-voltage activated currents, though less optimal for recording low-voltage activated currents (cf Karst et al, 2000 and Joëls et al, 2003). As it was found that at least the high-voltage activated L-type Ca-currents are very sensitive to corticosteroid receptor activation (Chameau et al, 2006), we preferred the use of the present pipette solution. Part of the electrodes also contained an intracellular dye (Alexa Fluor 594; Molecular Probes, The Netherlands); we did not observe any effect of this dye on the recorded currents (not shown), so that data obtained with and without the dye in the pipette could be pooled.

Under visible light the patch electrode was placed on the surface of the selected cell while applying positive pressure. By removing the pressure followed by a light suction, a gigaseal was established. Further suction disrupted the membrane, enabling whole cell recording. Cells were selected for recording if they displayed a pyramidal-shaped cell body located within the stratum pyramidale.

Whole cell Ca-currents were recorded under voltage clamp conditions, with an Axopatch 200B patch clamp amplifier (Axon Instruments, USA). Series resistance was compensated for approximately $70 \%$. Membrane capacitance was read directly off the capacitance compensation potentiometer on the patch amplifier, as described by others (Gibbs et al, 1997) and found by us to give reproducible values. Data acquisition was performed with PClamp (version 8.2) and analyzed with Clampfit (version 8.2). Currents were evoked by a protocol in which depolarizing pulses (200 ms duration) were applied to increasing voltage levels (varying between -80 and $0 \mathrm{mV}$, $10 \mathrm{mV}$ difference between steps) from a $3 \mathrm{~s}$ prepotential step at $-130 \mathrm{mV}$; the latter was introduced to remove all steady state inactivation. In all protocols, a period of $10 \mathrm{~s}$ was allowed between successive pulses to promote full extrusion of $\mathrm{Ca}$-ions which had entered upon depolarization. Routinely, off-line correction for linear leak current was applied as estimated from sequential depolarizing and hyperpolarizing voltage steps of $5 \mathrm{mV}$ and $50 \mathrm{~ms}$ duration at holding potential (Joëls and Karst, 1995); currents were not corrected, although, for the capacity transient introduced by the fluid column around the pipette.

\section{Data Analysis}

All data are expressed as mean \pm standard error of the mean (SEM). Data on the neuroendocrine parameters (body weight gain, adrenal weight, thymus weight and plasma corticosterone level) were analysed with a two-way ANOVA, with stress and RU 38486 treatment as the between-subjects factors. If overall significant differences were observed, post hoc comparison between appropriate groups was performed.

Data on the cell capacitance, currents at $-20 \mathrm{mV}$ and current density were statistically analyzed with a between subjects univariate analysis, with chronic stress, RU 38486 treatment and corticosterone administration as the three independent variables. Data on the Ca-current amplitude were analysed with a general linear model approach for repeated measures. In all cases, $p<0.05$ was considered to indicate a significant difference. If a significant effect of treatment was observed, data were subjected to a post hoc multiple comparison of the means.

\section{RESULTS \\ Effects of Chronic Stress and RU 38486 on Neuroendocrine Parameters}

Chronic unpredictable stress caused a significantly attenuated weight, compared to daily handling of animals (Table 1). The adrenal weight (absolute or expressed per $100 \mathrm{mg}$ bodyweight) was enhanced after chronic stress. Chronic stress only had an overall significant effect on the absolute thymus weight, not when thymus weight was expressed relative to body weight. Morning plasma corticosterone levels were low in all animals. Although the levels were on average slightly elevated after chronic stress, this difference did not reach significance $(p=0.13)$.

RU 38486 treatment by itself did not significantly affect the gain in body weight, thymus weight or morning plasma corticosterone concentration (Table 1). We also did not observe a significant interaction between stress and RU 38486: The effects of stress on bodyweight gain

Table I Effect of Chronic Stress and RU 38486 Treatment on Gain in Bodyweight during the 21 Days Stress/Handling Period (in g), on Adrenal and Thymus Weight (Absolute in grams; Relative in $\mathrm{g} / \mathrm{l} 00 \mathrm{~g}$ Bodyweight) and on Morning Plasma Corticosterone Levels (in $\mu \mathrm{g} / \mathrm{dl}$ )

Group (\# animals) $\Delta$ Body weight Adrenal weight (abs) Adrenal weight (rel) Thymus weight (abs) Thymus weight (rel) (CORT)

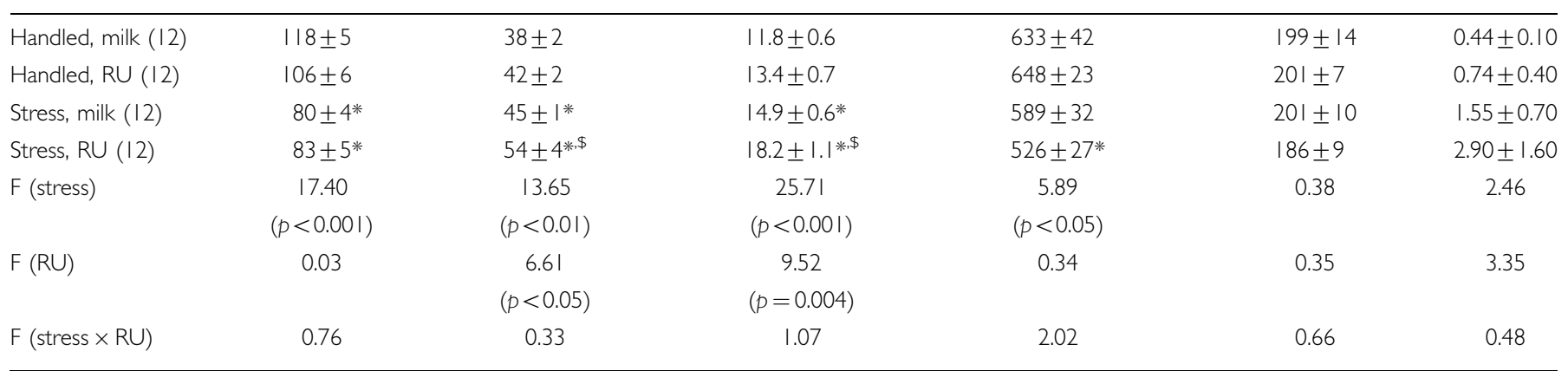

Data are expressed as mean \pm SEM. For statistical analysis, data were first subjected to a two-way analysis of variance (with stress and RU 38486 treatment as the between-subjects variables), followed by a post hoc comparison of the means. F-values are given at the bottom of the table.

${ }^{*} p<0.05$ compared to the corresponding handled control group; ${ }^{\$} p<0.05$ compared to the corresponding milk-injected group. 
and adrenal weight were not reversed by RU 38486 treatment on days 18-21. However, we did observe a significant overall effect of RU 38486 treatment on adrenal weight: adrenal weight (both absolute and relative to body weight) was highest in the chronically stressed rats receiving RU 38486.

\section{Effects of Chronic Stress, RU 38486 and Corticosterone Treatment on Ca-Current Amplitude}

Ca-currents were evoked and analyzed as shown in Figure 1. In all cells, we established the following characteristics: (1) the peak amplitude for each current trace; (2) the amplitude of the sustained component (ie, the average over the last $10 \mathrm{~ms}$ of the trace); (3) the current-voltage relationship for the peak and sustained current components; and (4) the current density for representative voltage steps (ie, the amplitude of the peak or sustained components at -30 , -20 , and $-10 \mathrm{mV}$ divided by the capacitance).

Figure 2 depicts the averaged current-voltage plots for the peak component of the $\mathrm{Ca}$-currents recorded in the present study. For reasons of clarity, the effects of stress and RU 38486 treatment in slices with low basal corticosterone levels (Figure 2a) are depicted separately from the effects of stress and in vitro corticosterone treatment (in rats not subjected to RU 38486; Figure 2b). For statistical analysis, though, we compared all eight experimental groups, applying a general linear model analysis for repeated measures. In this analysis, the recorded peak amplitude
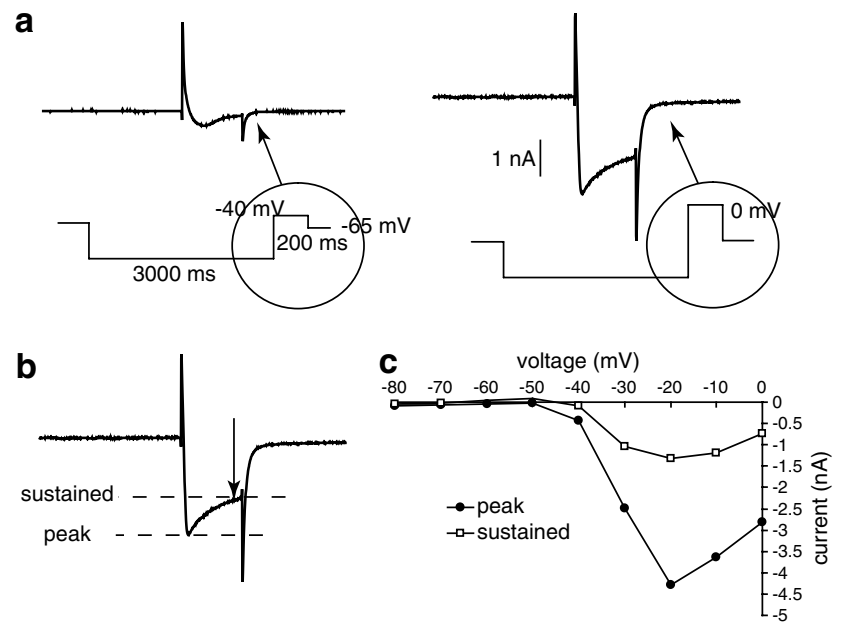

Figure I Typical examples of calcium currents evoked in a CAI hippocampal cell by depolarizing voltage steps of $200 \mathrm{~ms}$ duration (a). The left trace shows a current evoked by a step to $-40 \mathrm{mV}$, which mostly opens transient low-voltage activated channels. The inset below depicts the voltage protocol that was used to evoke the current shown in the figure; the encircled range corresponds to the time where the current trace shown on top was induced. The trace on the right shows a current evoked by a step to $0 \mathrm{mV}$. (b) Indicates how the peak and sustained components were determined. For the sustained component we calculated the averaged signal of the last $10 \mathrm{~ms}$ of each trace, as indicated by the downward arrow. In each cell complete current-voltage relationships for both the peak and sustained components were determined, as shown for an example in (c). The curves illustrate that the sustained component is smaller and activated at more depolarized $(>-30 \mathrm{mV})$ potentials than the peak of the current. in the range of -80 to $0 \mathrm{mV}$ was the dependent (within subjects) variable, while chronic stress/handling (1), RU38486/milk treatment, (2) and corticosterone/vehicle incubation, (3) represented the independent (betweensubjects) variables.

We observed a significant overall effect of chronic stress on the peak Ca-current amplitude $(\mathrm{F}(1,91)=4.44$, $p=0.038)$. There was no overall effect on Ca-current amplitude of treatment with RU $38486(\mathrm{~F}(1,91)=0.68$, $p=0.41)$ nor with corticosterone in vitro $(\mathrm{F}(1,91)=1.66$, $p=0.20)$. The overall interaction between chronic stress treatment and corticosterone incubation in vitro was nearly significant $(\mathrm{F}(1,91)=9.72, p=0.068)$. A very significant
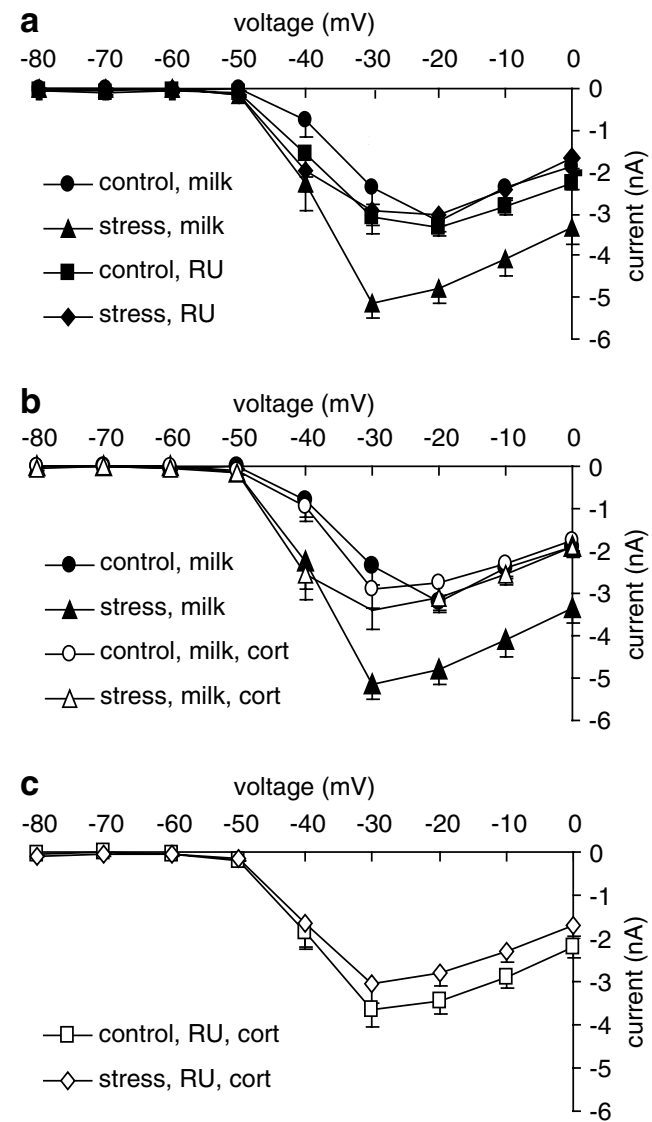

Figure 2 Averaged current-voltage plots (+SEM) for the peak component of the calcium currents in the various experimental groups. (a) Ca-current amplitude is increased in CAI hippocampal cells recorded one day after 21 days exposure to unpredictable stress $(n=13$ cells) compared to the handled control group $(n=9)$. Both groups received milk twice daily during days 17-21. Treatment of stressed rats during days 18 21 with the GR-antagonist RU $38486(n=13)$ results in currents that are comparable to those seen in the handled control group. Treatment of the controls with RU $38486(n=13)$ did not result in any change of the calcium currents. (b) Treatment of slices from handled control animals with corticosterone $(n=1 \mathrm{I})$ does not lead to an increase in the calcium current amplitude compared to the handled group treated with vehicle $(n=9)$. In slices from chronically stressed rats, currents recorded $1-4 \mathrm{~h}$ after corticosterone administration ( $n=13)$ were even found to be significantly reduced compared to the corresponding control group $(n=13)$. (c) Currents in corticosterone treated slices from animals injected with $\mathrm{RU}$ 38486 were small, both in the handled control group $(n=16)$ and the animals exposed to unpredictable stress $(n=11)$. Statistical analysis was performed over all groups and is described in detail in the Results section. 
overall interaction effect between chronic stress application and RU 38486 treatment was seen $(\mathrm{F}(1,91)=14.54$, $p<0.001$ ), indicating that the effect of chronic stress on Ca-current amplitude depended on the cotreatment with RU 38486. Analysis of variance over all experimental groups showed a significant difference between the eight experimental conditions $(\mathrm{F}(1,91)=4.16, p=0.001)$.

In agreement with these overall trends, post hoc multiple comparison of the means of the eight experimental groups over the entire voltage range revealed that peak currents recorded in vehicle-treated slices from chronically stressed rats were significantly larger than currents in vehicletreated slices from handled controls $(p<0.001$; Figure 2a). The stress-induced enhancement of the peak-current amplitude in vehicle-treated slices was fully normalized by a 4-days-treatment with RU 38486 ( $p<0.001$; Figure 2a), while RU 38486 treatment did not change Ca-current characteristics in the handled control group. Moreover, Ca-currents were significantly decreased after corticosterone treatment of slices from chronically stressed rats $(p=0.03)$, while they were not affected by corticosterone in the handled control group (Figure $2 \mathrm{~b}$ ). Corticosterone administration did not affect the Ca-current amplitude in slices from RU 38486 treated rats (regardless whether they had been stressed or not), similar to what was seen in milktreated handled controls (Figure 2c).

Very comparable data were observed for the sustained component of the current (data not shown). A highly significant overall interaction effect was observed for chronic stress and RU 38486 treatment $(F(1,88)=10.02$, $p=0.002)$, again indicating that sustained currents are differently affected by stress depending on the co-treatment with RU 38486. Chronic stress by itself did just not significantly affect the current $(\mathrm{F}(1,88)=3.72, p=0.057)$, while RU 38486 treatment alone or corticosterone incubation did not affect the current at all $(F(1,88)=0.033$ and 0.044 , respectively). Analysis of variance on all 8 experimental groups showed a significant difference between the various conditions $(\mathrm{F}(1,88)=3.34, p=0.003)$. Post hoc comparison of the means over the entire voltage range revealed that in vehicle-treated slices from chronically stressed rats the sustained current component was significantly $(p<0.001$ for the entire voltage range) enhanced compared to the handled control group. Co-treatment of chronically stressed rats with RU 38486 normalized the sustained Ca-current amplitude $(p<0.001$, compared to the milk-treated stressed group). The sustained current amplitude in chronically stressed rats (not receiving RU 38486) was significantly ( $p=0.005$ for the entire voltage range) reduced 1-4h after in vitro corticosterone treatment. Corticosterone was ineffective in slices from handled control rats.

Interestingly, we also observed a significant overall effect of chronic stress on cell capacitance $(\mathrm{F}(1,92)=9.02$; $p=0.003)$. Moreover, there was a strong interaction effect for capacitance between chronic stress and RU 38486 treatment $(\mathrm{F}(1,92)=6.29 ; p=0.014)$. Post hoc analysis demonstrated a significant and consistent increase $(p<0.05)$ in the capacitance after chronic stress, both after vehicle treatment $(p=0.003)$ and $1-4 \mathrm{~h}$ after corticosterone treatment $(p=0.03$; Table 2$)$. In the whole cell recording mode, capacitance is probably mostly determined by the
Table 2 Effect of Chronic Stress/RU 38486 Treatment on Capacitance and Current Density

Capacitance Peak current

Current density

\begin{tabular}{clll}
\hline Vehicle treatment & & & \\
Handling, milk & $26.9 \pm 2.0$ & $3.2 \pm 0.2$ & $12.3 \pm 1.0$ \\
Stress, milk & $35.6 \pm 2.6^{* * *}$ & $4.8 \pm 0.4^{*} * *$ & $13.8 \pm 0.9$ \\
Handling, RU & $31.8 \pm 1.4$ & $3.3 \pm 0.2$ & $10.9 \pm 1.0$ \\
Stress, RU & $32.9 \pm 1.8$ & $3.0 \pm 0.3^{\$}$ & $9.4 \pm 1.0^{\$}$
\end{tabular}

Corticosterone treatment

$\begin{array}{lllc}\text { Handling, milk } & 27.2 \pm 1.3 & 2.7 \pm 0.3 & 10.2 \pm 1.1 \\ \text { Stress, milk } & 33.5 \pm 2.0^{*} & 3.1 \pm 0.3^{\#} & 9.5 \pm 0.9^{\#} \\ \text { Handling, RU } & 30.7 \pm 2.0 & 3.4 \pm 0.5 & 10.8 \pm 1.0 \\ \text { Stress, RU } & 30.9 \pm 1.7 & 2.8 \pm 0.4 & 9.5 \pm 1.4\end{array}$

Chronic stress increases the capacitance (in pF; left) of CAI pyramidal cells compared to the handling procedure, both in vehicle treated hippocampal slices and in corticosterone treated slices. Consequently, while the mean peak current (in $\mathrm{nA}$ ) measured at a representative voltage step of $-20 \mathrm{mV}$ is increased after chronic stress compared to control handling (middle), the current density is not (right; in $\mathrm{nA} / \mathrm{pF}$ ). However, both the mean peak current and the current density are significantly reduced by $\mathrm{RU} 38486$ treatment during the last 4 days of the stress protocol in vehicle treated slices as well as by corticosterone treatment in vitro in combination with chronic stress.

All data represent the mean \pm SEM. For the number of cells: see legend of Figure 2. Statistical analysis was performed over all groups and is described in detail in the Results section. ${ }^{*} p<0.05$, $* * * 0.01$, chronic stress versus handling control; ${ }^{\$} p<0.01$, RU 38486 versus milk treated (in vivo); ${ }^{*} p<0.01$, corticosterone versus vehicle treated (in vitro).

cell surface near the patch pipette, that is the size of the soma and primary dendrite (Anderson et al, 2001). This could indicate that the enhanced current amplitude after chronic stress was caused by a larger surface close to the patch pipette, rather than an increased channel density. When the amplitude of the peak current component was divided by the capacitance (an index for current density) we no longer observed differences after chronic stress, as shown for a representative voltage step to $-20 \mathrm{mV}$ in Table 2. Comparable observations were carried out with respect to the current density for voltage steps to -30 and $-10 \mathrm{mV}$ (data not shown). It should be noted, though, that current density analyzed for voltage steps to -30 and $-10 \mathrm{mV}$ still tended $(p=0.072$ and 0.061 , respectively) towards an increase after chronic stress compared to the corresponding control group. Analysis of the sustained current component yielded very similar results; for this component too, the current density for voltage steps to -30 , -20 , or $-10 \mathrm{mV}$ was not different in the chronic stress groups $v s$ the controls $(p=0.089,0.190$, and 0.289 for -30 , -20 , and $-10 \mathrm{mV}$, respectively). The significant decreases in the peak current components caused either by RU 38486 treatment or by corticosterone treatment in vitro in the chronically stressed group were paralleled by a similar decrease in current density (Table 2), indicating that these two conditions alter Ca-influx in a different way than chronic stress per se. This was also observed for the sustained current component $(p<0.05$ for the two conditions and three voltage steps; data not shown). 


\section{Effect of Corticosterone in Naïve, Control Rats}

As shown in Figure $2 \mathrm{~b}$ and Table 2, CA1 cells in slices from handled control rats did not exhibit an increase in the amplitude of the Ca-current $1-4 \mathrm{~h}$ after a brief treatment with corticosterone $(p=0.95)$. This contrasts with earlier studies on tissue from younger, naïve rats or mice, where corticosterone was consistently found to enhance Cacurrent amplitude in CA1 cells (Kerr et al, 1992; Karst et al, 1994, 2000). The discrepancies might have been caused by two differences between the present and earlier experimental conditions: first, the present animals were slightly older in age. Effects of adrenalectomy (ie, absence of corticosterone) on Ca-current amplitude were found to depend on the age of the animal (Karst et al, 1997b); this might also play a role when corticosterone is added to the slice. Second, the control group for the chronically stressed rats were handled daily and also received milk via a gastrooesophageal tube during days $18-21$ of the handling protocol. Both manipulations might have caused mild stress to the animals, even though this was not obvious from any of the presently analyzed neuroendocrine parameters at day 22. To distinguish between these possibilities, we also tested the effect of in vitro corticosterone treatment on slices prepared from naive rats of the same age as the handled control group. As shown in Figure 3 for a representative

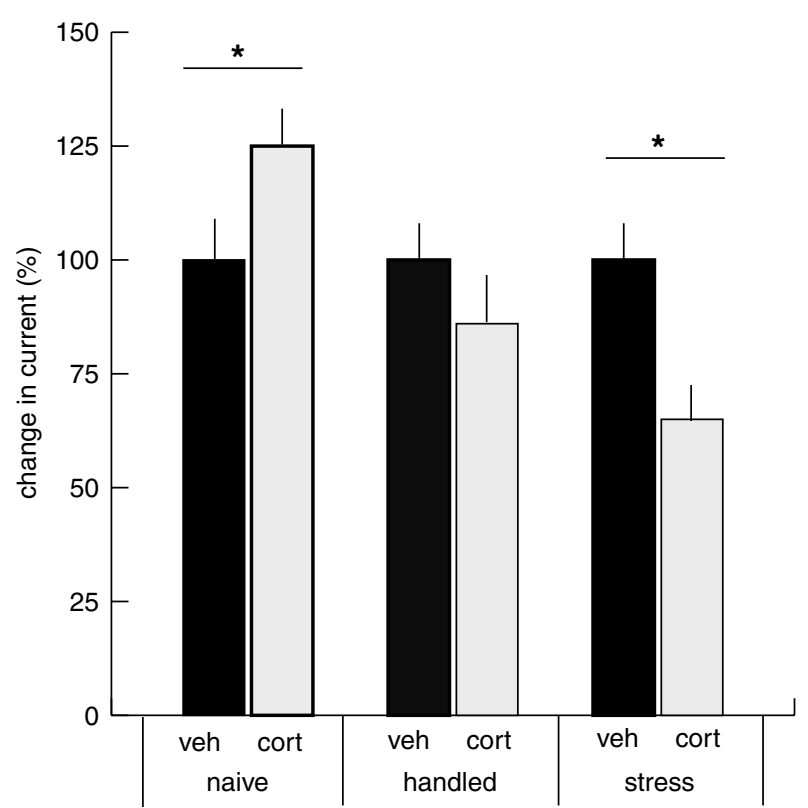

Figure 3 Ca-currents evoked in CAI neurons from naïve rats by a voltage step to $-20 \mathrm{mV}$ are significantly increased $1-4 \mathrm{~h}$ after a brief (20 min) administration of corticosterone in vitro (cort; $n=12$ cells), compared to the vehicle treated group (veh; $n=11$; left panel). The corticosterone dependent increase was no longer seen in slices prepared from rats that were handled for $2 \mathrm{I}$ days and treated with milk during days $|8-2|$ (middle). In rats subjected to $2 \mid$ days of unpredictable stress (in combination with milk treatment), corticosterone administration resulted in a significant reduction of the Ca-current amplitude (right). All data refer to the peak component of the currents. Vehicle-treated groups are represented by black bars, corticosterone treated groups by grey bars. To allow better comparison between the various conditions, the currents were normalized to the average of the vehicle treated group, for each set of data. The bars represent the mean + SEM. Statistical comparison for each set of results was performed with a Student's t-test ( $p<0.05)$. voltage step to $-20 \mathrm{mV}$ (currents normalized to the values in vehicle treated slices), CA1 neurons in slices from naïve animals indeed displayed the characteristic enhancement in Ca-current amplitude 1-4 h after brief (20 min) corticosterone administration in vitro (mean \pm SEM for the naïve, vehicle treated group: $-4.1 \pm 0.4 \mathrm{nA}$; naïve, corticosterone: $-5.2 \pm 0.3 \mathrm{nA} ; p=0.04)$.

\section{DISCUSSION}

The chronic unpredictable stress model in rats represents some of the risk factors for onset of major depression in humans. In the present study, we focused on two questions regarding this model: (i) we examined if 21 days of unpredictable stress affect Ca-currents in CA1 pyramidal neurons, a property that so far has not been studied after chronic stress; and (ii) if so, whether this can be normalized by 4-days treatment with the GR-antagonist RU 38486, a compound that was recently described to rapidly ameliorate symptoms of bipolar or psychotic depression in humans (Belanoff et al, 2001; Young et al, 2004; Simpson et al, 2005; Flores et al, 2006).

Our data unequivocally indicate that chronic stress - particularly under conditions that low levels of corticosterone circulate-enhances the total Ca-influx into CA1 pyramidal neurons. This situation is normalized by simultaneous treatment with RU 38486 during the last 4 days of the stress protocol. Capacitance was also enhanced after chronic stress, suggesting that the surface of the soma and primary dendrites rather than the density of calcium channels are increased.

\section{Chronic Stress Protocol}

Morning plasma corticosterone levels after chronic stress, although on average doubled, were not significantly elevated. This is in agreement with an earlier set of experiments where we used the same protocol (eg Alfarez et al, 2003; Van Riel et al, 2003). Previously we found a similar relative increase in the basal corticosterone level after chronic stress as well as considerable variability among animals. Consequently, significance was only attained in large cohorts of animals, that is not with limited group sizes as presently used (Verkuyl et al, 2004). Other studies that used unpredictable stress for seven (Ostrander et al, 2006) or 13 days (Chappell et al, 1986) also reported roughly a doubling of the morning plasma corticosterone levels, although in those cases data were less variable and significance was attained with fairly small group-sizes; the lower degree of variability may be due to the rat strain used (Sprague-Dawley) which was different from the one used in the present study (Wistar).

Although we did not observe a significant elevation of basal morning plasma corticosterone level one day after the last stress exposure, this does not preclude that differences may exist at other times of the diurnal rhythm. Indeed, our data clearly indicate that during the 21 days of unpredictable stress rats were exposed to elevated plasma corticosterone levels. Thus, in agreement with earlier studies (eg Herman et al, 1995; Alfarez et al, 2003), their gain in bodyweight was decreased and adrenal size - both absolute and relative to body weight - was increased. The absolute 
thymus weight was decreased after chronic stress; however, when expressed relative to the bodyweight - which is reduced after chronic stress - the thymus weight was not significantly changed, similar to what we saw in an earlier set of experiments (eg, Van Riel et al, 2003). This differs from what is found with exogenous administration of very high doses of corticosterone (eg, Karten et al, 1999) and may indicate that the increase in corticosteroid level after chronic unpredictable stress is relatively mild.

Interestingly, the change in bodyweight gain after chronic stress was not normalized by RU 38486 treatment. This most likely is caused by the fact that the most prominent change in bodyweight takes place during the first weeks of the chronic stress protocol and hence is not affected by RU 38486 treatment towards the end of the 3 weeks stress period. A certain degree of adaptation to repetitive stress is indeed known to occur over several weeks (Spencer and McEwen, 1990; Watanabe et al, 1992a; Magarinos and McEwen, 1995b), even with the variable protocol that we used in the current study (Chappell et al, 1986). Interestingly, adrenal weight was enhanced by RU 38486 in the chronically stressed group while corticosterone levels were not significantly affected. This is completely in line with an earlier study in naïve rats (Van Haarst et al, 1996). The latter study showed increased adrenal weight and enhanced in vitro adrenal responsiveness to ACTH after central RU 38486 infusion for several days; in vivo, though, only afternoon but not morning corticosterone levels were increased. Explanations for this apparent disparity supplied by the authors were that (1) GR blockade is not expected to play a major role in the morning, when mostly MRs are activated; and (2) that GR blockade could potentiate the sympathetic outflow which is known to enhance adrenal activity in the afternoon (Nicholson et al, 1985).

\section{Effect of Chronic Stress on Ca-Currents}

The fact that chronic stress affects cell properties in the CA1 area is not unprecedented. For instance, a similar protocol was earlier found to impair LTP (Alfarez et al, 2003) and to suppress responses to serotonin in the CA1 region (Van Riel et al, 2003). In other brain areas too, effects of 21 days of stress - restraint, social, unpredictable or other types of stress - on cell characteristics have been described (see for reviews McEwen and Chattarji (2004), Joëls et al (2004)). Of these, the morphological changes of pyramidal neurons in the hippocampal CA3 area (Watanabe et al, 1992a; Magarinos et al, 1996) and basolateral amygdala are most prominently documented (Vyas et al, 2002; Fuchs et al, 2006). Yet, many more effects including changes in glutamate responses in the CA3 region (Kole et al, 2002, 2004) and dentate gyrus (Karst and Joëls, 2003) as well as regarding inhibitory control of parvocellular neurons in the hypothalamus (Verkuyl et al, 2004) have been found.

While the present effects on Ca-current amplitude are thus in line with earlier cellular observations, some care with the interpretation of changes in Ca-current properties is required. In our study, Ca-currents were recorded in the whole cell configuration which in CA1 neurons is known to suffer from space clamp problems. Bold statements about effects of chronic stress on for example voltage dependency or kinetic properties therefore cannot be made. Still, the present data strongly indicate that the current amplitude is increased by chronic stress. In this way, chronic stress could make CA1 cells more vulnerable to additional challenges causing lasting depolarizations, such as during epilepsy or ischemia.

Interestingly, chronic stress was associated with a larger cell capacitance. Current density for voltage steps to -30 , -20 , or $-10 \mathrm{mV}$ was not significantly changed after chronic stress. This suggests that the enhanced Ca-current amplitude after chronic stress is at least partly attributable to an enhanced cell surface close to the recording pipette (Anderson et al, 2001), rather than an enhanced conductance or density of channels. This might involve trophic factors which are known to be altered in expression by chronic stress (Smith et al, 1995). If so, the present data could point to morphological alterations in the CA1 area unlike those previously described after chronic restraint stress for pyramidal neurons in the CA3 region (Watanabe et al, 1992a,b) and the prefrontal cortex (Cook and Wellman, 2004; Radley et al, 2004) but reminiscent of the basolateral amygdala (Vyas et al, 2002). Notably, the Ca-current changes observed after RU 38486 treatment or corticosterone administration to slices from stressed rats (see also below) were not accompanied by differences in capacitance. This may signify that these treatments alter currents and possibly morphology in a different way than chronic stress.

Unexpectedly, in vitro administration of corticosterone, at a dose that supposedly activates GRs in addition to MRs (Reul and de Kloet, 1985), did not result in larger Cacurrent amplitudes in the handled control group. This contrasts with the increased amplitude seen in younger, naïve rats (Kerr et al, 1992; Karst et al, 1994, 2000). Currents in naïve rats of the same age, however, did show enhancement after corticosterone treatment. The data suggest that daily handling in combination with gastrooesophageal administration of milk for 4 days alters the responsivity of CA1 neurons to in vitro corticosterone administration. While naive rats show an enhanced response to corticosterone, handled rats become unresponsive, whereas chronically stressed rats even show a reduced Ca-current amplitude. A comparable importance of the animals' background for the direction of corticosteroid effects was earlier observed in apolipoprotein-E knockout $v s$ wildtype mice (Grootendorst et al, 2001). Similarly, preliminary data show that corticosterone decreases longterm potentiation in rats which received a high degree of maternal care while it enhances long-term potentiation in the offspring of low-care mothers (Champagne et al, 2006). The present data thus underline that the 'control' procedure of handling may result in a different condition than when rats are left mostly undisturbed. This has been reported earlier in a study showing that corticosterone release after an acute stress is blunted in adult, handled animals compared to naïve animals (Gadek-Michalska and Bugajski, 2003); the importance of handling, though, is especially documented for the sensitive early postnatal period (Meaney, 2001; Sanchez et al, 2001).

\section{RU 38486 Treatment}

The enhancement of Ca-current amplitude after chronic stress, under basal corticosterone conditions, was not seen 
in animals treated during days 18-21 with the GRantagonist RU 38486, given orally before stress exposure. In view of the short half-life time of RU 38486, particularly in rats (1-2 $\mathrm{h}$ in plasma; see Heikinheimo and Kekkonen, 1993; Heikinheimo et al, 1994), it is unlikely that this effect was caused by the antagonist still circulating on the day of the experiment, that is $>16 \mathrm{~h}$ after the last treatment. Rather, RU 38486 seems to reverse the effects of earlier stress exposure (during days 1-17) or to block putative effects developing during the last days of the stress protocol.

Normalizing effects on morphological parameters of CA3 neurons after 21 days of restraint stress were earlier observed after treatment with the antiepileptic drug phenytoin (Watanabe et al, 1992b), with benzodiazepines (Magarinos et al, 1999) or an NMDA-receptor antagonist (Magarinos and McEwen, 1995a). Similarly, the antidepressant tianeptine, which decreases the bio-availability of serotonin (Whitton et al, 1991) but also affects glutamate transmission (Kole et al, 2002), has been reported to prevent dendritic retraction of CA3 pyramidal neurons and the enhanced AMPA-receptor mediated responses of these cells after restraint stress (Magarinos et al, 1999; Kole et al, 2002). Tianeptine was also found to prevent the impairment of neurogenesis in the dentate gyrus after chronic restraint stress (Czeh et al, 2001). Interestingly, tianeptine as well as other antidepressants by themselves already affect characteristics of CA3 or dentate cells, in the direction opposite to that caused by chronic stress (Malberg et al, 2000; Kole et al, 2002; Santarelli et al, 2003). This suggests that their normalizing effects do not directly interfere with the effects of chronic stress, but rather compensate these effects possibly through alternative pathways. By contrast, we found a specific interaction between chronic stress and RU 38486 treatment, indicating that the treatment interferes directly with the consequences of chronic stress exposure: RU 38486 treatment alone is ineffective in handled controls, but prevents or reverses the enhanced Ca-current amplitude seen after chronic stress. Whether or not RU 38486 prevents/reverses the effects of chronic stress at the level of CA1 pyramidal cell signaling or by changing the surrounding of these cells (including their input) is presently unclear. It should be realized that the 4-daystreatment not only affects the CA1 area but also brain regions projecting to this area, as well as peripheral organs.

The time course of the effects by RU 38486 on Ca-currents is comparable to that of the ameliorating effects by this compound seen in patients with psychotic or bipolar depression (Belanoff et al, 2001; Young et al, 2004; Simpson et al, 2005; Flores et al, 2006). Thus, in individuals with psychotic depression it was found that significantly more patients in the group treated for 4-7 days with RU 38486 showed a $50 \%$ or greater decline on a positive symptom subscale which serves as an index of psychotic symptoms (Belanoff et al, 2001; Flores et al, 2006). In a cohort with bipolar depression, improvement in spatial working memory performance, verbal fluency as well as beneficial effects on mood was observed upon 1 week of treatment with RU 38486 (Young et al, 2004). The present data in rats are among the first to show that such treatment can indeed have normalizing effects on single cell properties in brain. This and other studies investigating the effects of temporary treatment with RU 38486 against a background of HPA- hyperactivity on properties of brain cells may thus form a mechanistic underpinning of the clinical effects of this compound.

\section{ACKNOWLEDGEMENTS}

We thank Maaike van de Mark for the radioimmunoassay of corticosterone and Drs Emiel van Loon and Anda van Stegeren for statistical advice.

\section{CONFLICT OF INTEREST}

MJ has no financial interests to disclose but her spouse (antenuptial contract) is an advisor and has stocks of Corcept.

\section{REFERENCES}

Alfarez DN, Joëls M, Krugers HJ (2003). Chronic unpredictable stress impairs long-term potentiation in rat hippocampal CA1 area and dentate gyrus in vitro. Eur J Neurosci 17: 1928-1934.

Anderson RL, Jobling P, Gibbins IL (2001). Development of electrophysiological and morphological diversity in autonomic neurons. J Neurophysiol 86: 1237-1251.

Belanoff JK, Flores BH, Kalezhan M, Sund B, Schatzberg AF (2001). Rapid reversal of psychotic depression using mifepristone. J Clin Psychopharmacol 21: 516-521.

Berton O, Nestler EJ (2006). New approaches to antidepressant drug discovery: beyond monoamines. Nat Rev Neurosci 7: 137-151.

Brown ES, Varghese FP, McEwen BS (2004). Association of depression with medical illness: does cortisol play a role? Biol Psychiatry 55: 1-9.

Chameau P, Qin Y, Spijker S, Smit AB, Joëls M (2006). Glucocorticoids specifically enhance L-type calcium current amplitude and affect calcium channel subunit expression in the mouse hippocampus. J Neurophysiol, in press.

Champagne DL, De Kloet E, Meaney M, Joels M, Krugers H (2006). Maternal care and hippocampal plasticity. Soc Neurosci (Atlanta, GA) (abstract 562.1).

Chappell PB, Smith MA, Kilts CD, Bissette G, Ritchie J, Anderson C et al (1986). Alterations in corticotropin-releasing factor-like immunoreactivity in discrete rat brain regions after acute and chronic stress. J Neurosci 6: 2908-2914.

Conrad CD (2006). What is the functional significance of chronic stress-induced CA3 dendritic retraction within the hippocampus? Behav Cogn Neurosci Rev 5: 41-60.

Cook SC, Wellman CL (2004). Chronic stress alters dendritic morphology in rat medial prefrontal cortex. J Neurobiol 60: 236-248.

Cullinan WE, Wolfe TJ (2000). Chronic stress regulates levels of mRNA transcripts encoding beta subunits of the GABA(A) receptor in the rat stress axis. Brain Res 887: 118-124.

Czeh B, Michaelis T, Watanabe T, Frahm J, de Biurrun G, van Kampen $\mathrm{M}$ et al (2001). Stress-induced changes in cerebral metabolites, hippocampal volume, and cell proliferation are prevented by antidepressant treatment with tianeptine. Proc Natl Acad Sci USA 98: 12796-12801.

De Kloet ER, Joëls M, Holsboer F (2005). Stress and the brain: from adaptation to disease. Nat Rev Neurosci 6: 463-475.

Flores BH, Kenna H, Keller J, Solvason HB, Schatzberg AF (2006). Clinical and biological effects of mifepristone treatment for psychotic depression. Neuropsychopharmacology 31: 628-636.

Fuchs E, Flugge G, Czeh B (2006). Remodeling of neuronal networks by stress. Front Biosci 11: 2746-2758. 
Gadek-Michalska A, Bugajski J (2003). Repeated handling, restraint, or chronic crowding impair the hypothalamic-pituitary-adrenocortical response to acute restraint stress. J Physiol Pharmacol 54: 449-459.

Gibbs JW, Sombati S, De Lorenzo RJ, Coulter DA (1997). Physiological and pharmacological alterations in postsynaptic GABA(A) receptor function in a hippocampal culture model of chronic spontaneous seizures. J Neurophysiol 77: 2139-2152.

Grootendorst J, de Kloet ER, Dalm S, Oitzl MS (2001). Reversal of cognitive deficit of apolipoprotein $\mathrm{E}$ knockout mice after repeated exposure to a common environmental experience. Neuroscience 108: 237-247.

Heikinheimo O, Kekkonen R (1993). Dose-response relationships of RU 486. Ann Med 25: 71-76.

Heikinheimo O, Pesonen U, Huupponen R, Koulu M, Lahteenmaki $P$ (1994). Hepatic metabolism and distribution of mifepristone and its metabolites in rats. Hum Reprod 9(Suppl 1): 40-46.

Herman JP, Adams D, Prewitt C (1995). Regulatory changes in neuroendocrine stress-integrative circuitry produced by a variable stress paradigm. Neuroendocrinology 61: 180-190.

Holsboer F (2000). The corticosteroid receptor hypothesis of depression. Neuropsychopharmacology 23: 477-501.

Ising M, Kunzel HE, Binder EB, Nickel T, Modell S, Holsboer F (2005). The combined dexamethasone/CRH test as a potential surrogate marker in depression. Prog Neuropsychopharmacol Biol Psychiatry 29: 1085-1093.

Joëls M (2001). Corticosteroid actions in the hippocampus. J Neuroendocrinol 13: 657-669.

Joëls M, Karst H (1995). Effects of estradiol and progesterone on voltage-gated calcium and potassium conductances in rat CA1 hippocampal neurons. J Neurosci 15: 4289-4297.

Joëls M, Karst H, Alfarez D, Heine VM, Qin Y, van Riel E et al (2004). Effects of chronic stress on structure and cell function in rat hippocampus and hypothalamus. Stress 7: 221-231.

Joëls M, Velzing E, Nair S, Verkuyl JM, Karst H (2003). Acute stress increases calcium current amplitude in rat hippocampus: temporal changes in physiology and gene expression. Eur $J$ Neurosci 18: 1315-1324.

Karst H, Joëls M (2003). Effect of chronic stress on synaptic currents in rat hippocampal dentate gyrus neurons. $J$ Neurophysiol 89: 625-633.

Karst H, Karten YJ, Reichardt HM, de Kloet ER, Schütz G, Joëls M (2000). Corticosteroid actions in hippocampus require DNA binding of glucocorticoid receptor homodimers. Nat Neurosci 3: 977-978.

Karst H, de Kloet ER, Joëls M (1997a). Effect of ORG 34116, a corticosteroid receptor antagonist, on hippocampal $\mathrm{Ca} 2+$ currents. Eur J Pharmacol 339: 17-26.

Karst H, Wadman WJ, Joëls M (1994). Corticosteroid receptordependent modulation of calcium currents in rat hippocampal CA1 neurons. Brain Res 649: 234-242.

Karst H, Werkman TR, Struik M, Bosma A, Joëls M (1997b). Effects of adrenalectomy on $\mathrm{Ca} 2+$ currents and $\mathrm{Ca} 2+$ channel subunit mRNA expression in hippocampal CA1 neurons of young rats. Synapse 26: 155-164.

Karten YJ, Nair SM, van Essen L, Sibug R, Joëls M (1999). Longterm exposure to high corticosterone levels attenuates serotonin responses in rat hippocampal CA1 neurons. Proc Natl Acad Sci USA 96: 13456-13461.

Kerr DS, Campbell LW, Thibault O, Landfield PW (1992). Hippocampal glucocorticoid receptor activation enhances voltage-dependent $\mathrm{Ca} 2+$ conductances: relevance to brain aging. Proc Natl Acad Sci USA 89: 8527-8531.

Kole MH, Czeh B, Fuchs E (2004). Homeostatic maintenance in excitability of tree shrew hippocampal CA3 pyramidal neurons after chronic stress. Hippocampus 14: 742-751.

Kole MH, Swan L, Fuchs E (2002). The antidepressant tianeptine persistently modulates glutamate receptor currents of the hippocampal CA3 commissural associational synapse in chronically stressed rats. Eur J Neurosci 16: 807-816.

Magarinos AM, Deslandes A, McEwen BS (1999). Effects of antidepressants and benzodiazepine treatments on the dendritic structure of CA3 pyramidal neurons after chronic stress. Eur J Pharmacol 371: 113-122.

Magarinos AM, McEwen BS (1995a). Stress-induced atrophy of apical dendrites of hippocampal CA3c neurons: involvement of glucocorticoid secretion and excitatory amino acid receptors. Neuroscience 69: 89-98.

Magarinos AM, McEwen BS (1995b). Stress-induced atrophy of apical dendrites of hippocampal CA3c neurons: comparison of stressors. Neuroscience 69: 83-88.

Magarinos AM, McEwen BS, Flugge G, Fuchs E (1996). Chronic psychosocial stress causes apical dendritic atrophy of hippocampal CA3 pyramidal neurons in subordinate tree shrews. J Neurosci 16: 3534-3540.

Malberg JE, Eisch AJ, Nestler EJ, Duman RS (2000). Chronic antidepressant treatment increases neurogenesis in adult rat hippocampus. J Neurosci 20: 9104-9110.

McEwen BS (1999). Stress and hippocampal plasticity. Annu Rev Neurosci 22: 105-122.

McEwen BS, Chattarji S (2004). Molecular mechanisms of neuroplasticity and pharmacological implications: the example of tianeptine. Eur Neuropsychopharmacol 14: S497-S502.

McEwen BS, Wingfield JC (2003). The concept of allostasis in biology and biomedicine. Horm Behav 43: 2-15.

Meaney MJ (2001). Maternal care, gene expression, and the transmission of individual differences in stress reactivity across generations. Annu Rev Neurosci 24: 1161-1192.

Nair SM, Werkman TR, Craig J, Finnell R, Joëls M, Eberwine JH (1998). Corticosteroid regulation of ion channel conductances and mRNA levels in individual hippocampal CA1 neurons. J Neurosci 18: 2685-2696.

Nemeroff CB, Vale WW (2005). The neurobiology of depression: inroads to treatment and new drug discovery. J Clin Psychiatry 66(Suppl 7): 5-13.

Ni YG, Gold SJ, Iredale PA, Terwillger RZ, Duman RS, Nestler EJ (1999). Region-specific regulation of RGS4 (Regulator of G-protein-signaling protein type 4) in brain by stress and glucocorticoids: in vivo and in vitro studies. J Neurosci 19: 3674-3680.

Nicholson S, Lin J-H, Mahmoud S, Campbell E, Gillham B, Jones M (1985). Diurnal variations in responsiveness of the hypothalamo-pituitary-adrenal axis of the rat. Neuroendocrinology 40: 217-224.

Ostrander MM, Ulrich-Lai YM, Choi DC, Richtand NM, Herman JP (2006). Hypoactivity of the hypothalamo-pituitary-adrenocortical axis during recovery from chronic variable stress. Endocrinology 147: 2008-2017.

Radley JJ, Sisti HM, Hão J, Rocher AB, McCall T, Hof PR et al (2004). Chronic behavioral stress induces apical dendritic reorganization in pyramidal neurons of the medial prefrontal cortex. Neuroscience 125: 1-6.

Ribeiro SC, Tandon R, Grunhaus L, Greden JF (1993). The DST as a predictor of outcome in depression: a meta-analysis. Am J Psychiatry 150: 1618-1629.

Reul JM, de Kloet ER (1985). Two receptor systems for corticosterone in rat brain: microdistribution and differential occupation. Endocrinology 117: 2505-2511.

Sanchez MM, Ladd CO, Plotsky PM (2001). Early adverse experience as a developmental risk factor for later psychopathology: evidence from rodent and primate models. Dev Psychopathol 13: 419-449.

Santarelli L, Saxe M, Gross C, Surget A, Battaglia F, Dulawa S et al (2003). Requirement of hippocampal neurogenesis for the behavioral effects of antidepressants. Science 301: 805-809. 
Sergeyev V, Fetissov S, Mathe AA, Jimenez PA, Bartfai T, Mortas P et al (2005). Neuropeptide expression in rats exposed to chronic mild stresses. Psychopharmacology 178: 115-124.

Simpson GM, El Sheshai A, Loza N, Kingsbury SJ, Fayek M, Rady A et al (2005). An 8-week open-label trial of a 6-day course of mifepristone for the treatment of psychotic depression. J Clin Psychiatry 66: 598-602.

Smith MA, Makino S, Kvetnansky R, Post RM (1995). Stress and glucocorticoids affect the expression of brain-derived neurotrophic factor and neurotrophin-3 mRNAs in the hippocampus. J Neurosci 15: 1768-1777.

Spencer RL, McEwen BS (1990). Adaptation of the hypothalamicpituitary-adrenal axis to chronic ethanol stress. Neuroendocrinology 52: 481-489.

Van Haarst AD, Oitzl MS, Workel JO, de Kloet ER (1996). Chronic brain glucocorticoid receptor blockade enhances the rise in circadian and stress-induced pituitary-adrenal activity. Endocrinology 137: 4935-4943.

Van Riel E, Meijer OC, Steenbergen PJ, Joëls M (2003). Chronic unpredictable stress causes attenuation of serotonin responses in cornu ammonis 1 pyramidal neurons. Neuroscience 120: 649-658.

Van Steensel B, van Binnendijk EP, Hornsby CD, van der Voort HT, Krozowski ZS, de Kloet ER et al (1996). Partial colocalization of glucocorticoid and mineralocorticoid receptors in discrete compartments in nuclei of rat hippocampus neurons. J Cell Sci 109: 787-792.
Verkuyl JM, Hemby SE, Joëls M (2004). Chronic stress attenuates GABAergic inhibition and alters gene expression of parvocellular neurons in rat hypothalamus. Eur J Neurosci 20: 1665-1673.

Vyas A, Mitra R, Shankaranarayana rao BS, Chattarji S (2002). Chronic stress induces contrasting patterns of dendritic remodeling in hippocampal and amygdaloid neurons. J Neurosci 22: 6810-6818.

Watanabe Y, Gould E, McEwen BS (1992a). Stress induces atrophy of apical dendrites of hippocampal CA3 pyramidal neurons. Brain Res 588: 341-345.

Watanabe Y, Gould E, Cameron HA, Daniels DC, McEwen BS (1992b). Phenytoin prevents stress-and corticosterone-induced atrophy of CA3 pyramidal neurons. Hippocampus 2: 431-435.

Whitton PS, Sama GS, O'Connell MT, Curzon G (1991). The effect of the novel antidepressant tianeptine on the concentration of 5-hydroxytryptamine in rat hippocampal dialysates in vivo. Neuropharmacology 30: 1-4.

Young AH, Gallagher P, Watson S, Del-Estal D, Owen BM, Nicol Ferrier I (2004). Improvements in neurocognitive function and mood following adjunctive treatment with mifepristone (RU-486) in bipolar disorder. Neuropsychopharmacology 29: $1538-1545$.

Zobel AW, Nickel T, Sonnatag A, Uhr M, Holsboer F, Ising M (2001). Cortisol response in the combined dexamethasone/CRH test as predictor of relapse in patients with remitted depression. A prospective study. J Psychiatr Res 35: 83-94. 